\title{
Breeding for soil-borne pathogen resistance impacts active rhizosphere microbiome of common bean
}

\author{
Lucas William Mendes $\mathbb{(}^{1,2} \cdot$ Rodrigo Mendes $\mathbb{B}^{3} \cdot$ Jos M. Raaijmakers ${ }^{2,4} \cdot$ Siu Mui Tsai ${ }^{1}$
}

Received: 27 April 2018 / Revised: 13 June 2018 / Accepted: 15 June 2018 / Published online: 17 July 2018

(c) International Society for Microbial Ecology 2018

\begin{abstract}
Over the past century, plant breeding programs have substantially improved plant growth and health, but have not yet considered the potential effects on the plant microbiome. Here, we conducted metatranscriptome analysis to determine if and how breeding for resistance of common bean against the root pathogen Fusarium oxysporum (Fox) affected gene expression in the rhizobacterial community. Our data revealed that the microbiome of the Fox-resistant cultivar presented a significantly higher expression of genes associated with nutrient metabolism, motility, chemotaxis, and the biosynthesis of the antifungal compounds phenazine and colicin V. Network analysis further revealed a more complex community for Fox-resistant cultivar and indicated Paenibacillus as a keystone genus in the rhizosphere microbiome. We suggest that resistance breeding in common bean has unintentionally co-selected for plant traits that strengthen the rhizosphere microbiome network structure and enrich for specific beneficial bacterial genera that express antifungal traits involved in plant protection against infections by root pathogens.
\end{abstract}

The rhizosphere microbiome significantly impacts plant growth, development and resistance against soil-borne pathogens [1]. Plants shape their rhizosphere microbiome through exudates that stimulate or repress the abundance of specific microbial groups [2]. In return, rhizosphere microbes provide a range of ecosystem services for the plant, such as nutrient acquisition [3], abiotic stress tolerance [4], and protection against pathogens either directly via antagonism or indirectly via induction of systemic resistance [5, 6]. Recent studies further indicated that plant breeding for disease resistance has affected

Electronic supplementary material The online version of this article (https://doi.org/10.1038/s41396-018-0234-6) contains supplementary material, which is available to authorized users.

Lucas William Mendes

lucaswmendes@gmail.com

1 Cell and Molecular Biology Laboratory, Center for Nuclear Energy in Agriculture CENA, University of Sao Paulo USP, Piracicaba, SP 13416-000, Brazil

2 Departament of Microbial Ecology, Netherlands Institute of Ecology NIOO-KNAW, Wageningen 6708 PB, The Netherlands

3 Embrapa Meio Ambiente, Jaguariuna 18020-000, Brazil

4 Institute of Biology, Leiden University, Leiden, The Netherlands microbiome assembly in the rhizosphere [7, 8]. Considering that the rhizosphere microbiome provides a first line of defense for plants against pathogen invasion, we investigated if and how breeding of common bean (Phaseolus vulgaris) to the soil-borne pathogen Fusarium oxysporum f. sp. phaseoli (Fox) affected gene expression in the rhizosphere microbiome. In our previous study [7], using $16 \mathrm{~S}$ rRNA and metagenome sequencing, we showed that beneficial bacterial groups such as Pseudomonas and Bacillus, and genes involved in the biosynthesis of phenazines and rhamnolipids were more abundant in the rhizosphere of Fox-resistant bean cultivar. Whether these bacterial genera are active and if the enriched genes are actually expressed in the rhizosphere of the Foxresistant cultivar remains unclear. To further investigate the biological relevance of these DNA-based descriptive analyses, we set out a metatranscriptome analysis (RNAbased) to test if these and other putative bacterial genes are differentially expressed in the rhizosphere of the Foxresistant cultivar as compared to the Fox-susceptible. Here, both cultivars were grown under the same controlled conditions in a greenhouse experiment and metatranscriptome analysis was used to assess the active microbial taxa and functions in the rhizosphere of two common beans with contrasting levels of resistance to the soil-borne pathogen. 
(A)

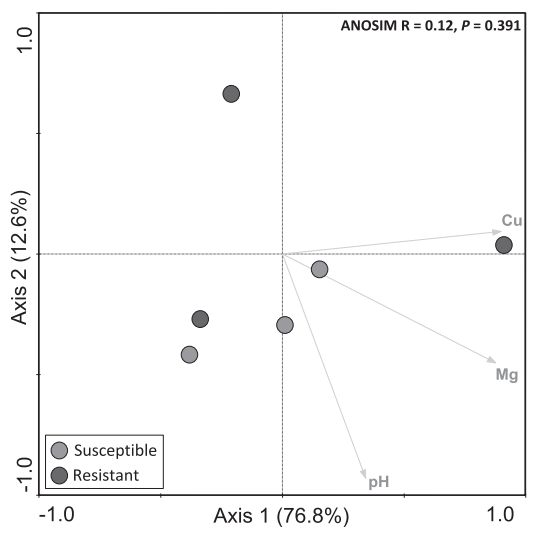

(C)
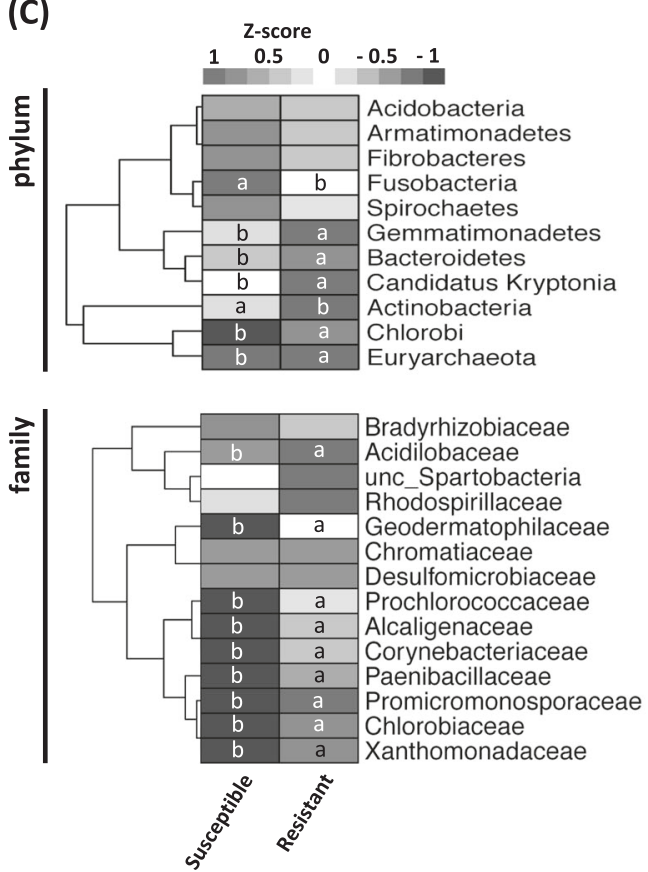

Fig. 1 Structure and composition of the microbial communities in the rhizosphere of two common bean cultivars with contrasting levels of resistance to $F$. oxysporum. a Redundancy analysis (RDA) performed on taxonomic profile (genus level) and environmental characteristics. Arrows indicate correlation between environmental parameters and community structure. Only significant correlations evaluated via the Monte Carlo permutation test $(P<0.05)$ are shown; b Principal component analysis (PCA) based on functional profile (SEED database). c Heatmap showing the differential abundance of

Our results showed high microbial activity in the rhizosphere of both cultivars, with parameters such as $\mathrm{pH}$ $(F=3.81, P<0.05)$, magnesium $(F=6.30, P<0.005)$, and cupper $(F=6.23, P<0.005)$ correlated with the overall community structure (Fig. 1a and Supplementary Table 1). Although the resolution obtained in taxonomical classification from the metatranscriptomic data did not show distinct communities between the two cultivars, the functional profile revealed a clear cultivar-specific clustering
(B)

\section{Function}

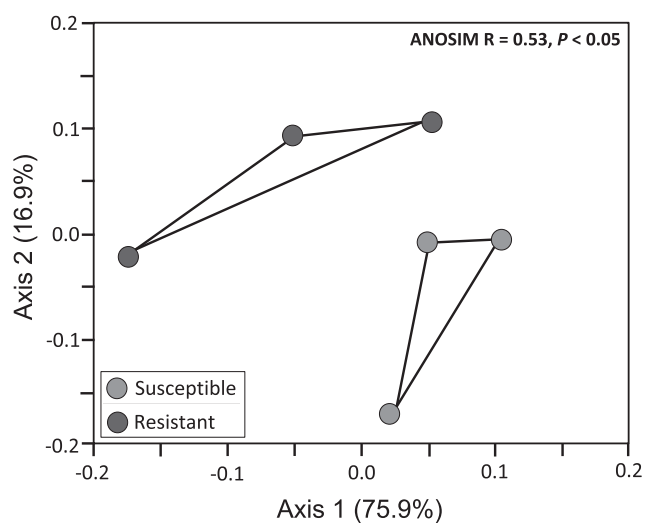

(D)
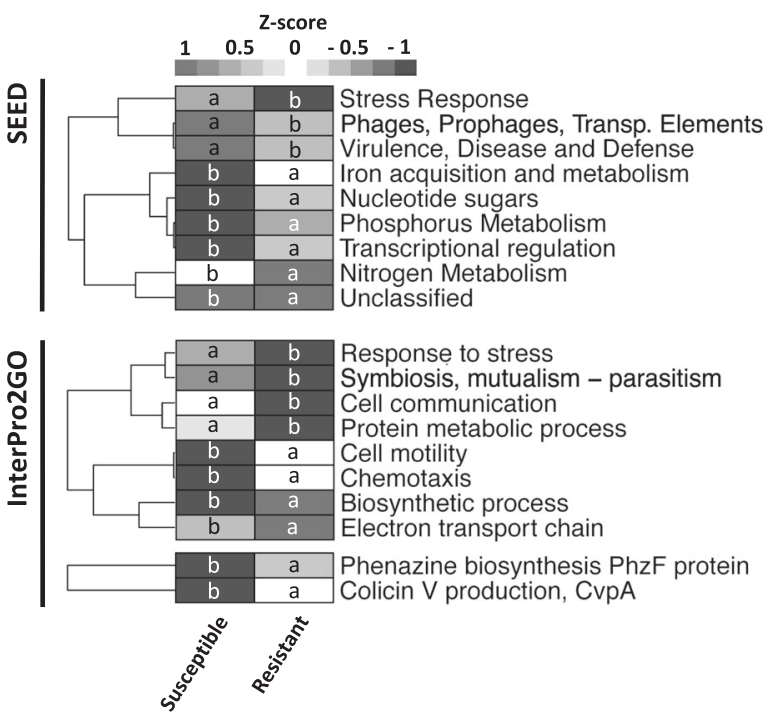

phylum and family and $\mathbf{d}$ heatmap for functional categories. The color key relates the heatmap colors to the standard score (z-score), i.e., the deviation from row mean in units of standard deviations above or below the mean. Different lower case letters refer to significant differences between the treatments based on Welch's $t$-test with Benjamini-Hochberg correction $(P<0.05)$. Significant clusters (ANOSIM, $P<0.05$ ) are indicated by lines in the graph B (color figure online)

of the samples (Fig. 1b). For both cultivars, the rhizosphere presented high activity of several taxa known as plant growth-promoting bacteria, such as the families Bradyrhizobiaceae, Rhizobiaceae, Rhodospirillaceae (Fig. 1c). Comparing the contrasting bean cultivars, we found higher activity of taxa affiliated to Paenibacillaceae in the Foxresistant rhizosphere. Several members of this bacterial family are well known for the production of antibiotics with inhibitory activity against phytopathogenic fungi [9]. 
(A) Bacterial Family

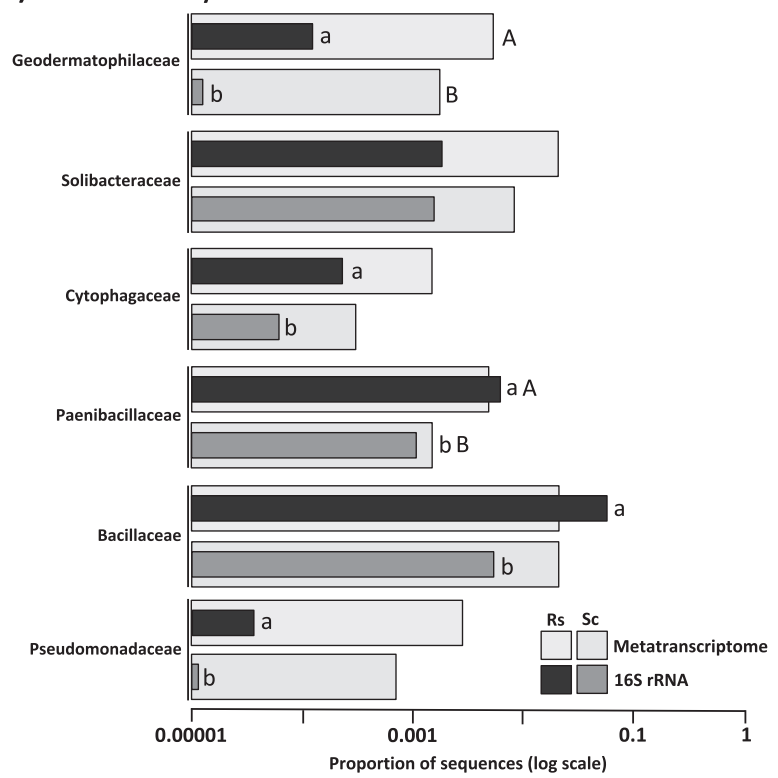

(C)

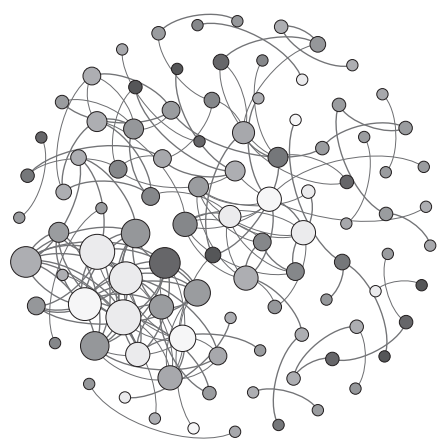

Susceptible

IAC Alvorada
(B) Functions
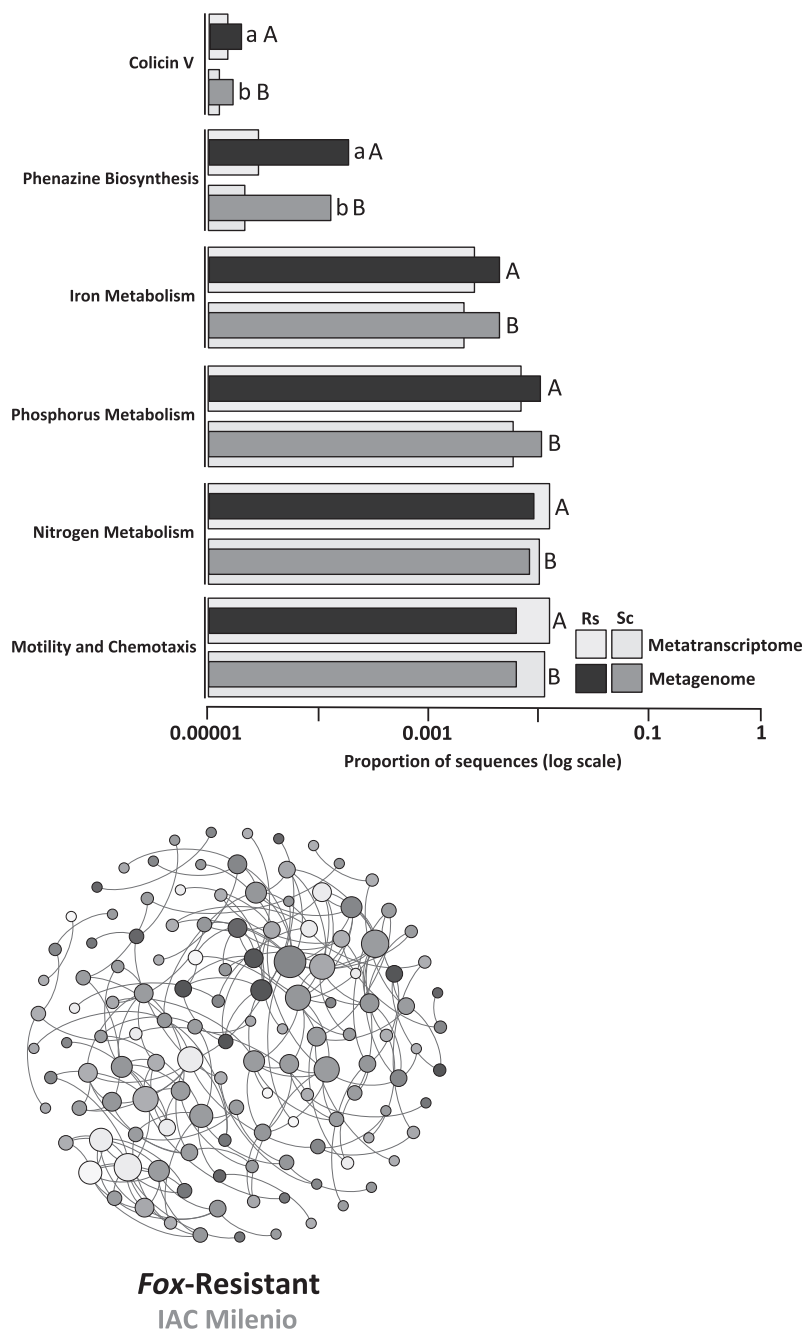

IAC Milenio

Acidobacteria OActinobacteria $\bigcirc$ Bacteroidetes $O$ chloroflexi OFirmicutes $\bigcirc$ Alphaproteobacteria $\bigcirc$ Betaproteobacteria

OGammaproteobacteria ODeltaproteobacteria OOthers

Fig. 2 a Taxonomic and $\mathbf{b}$ functional comparison between metatranscriptome data from this study and DNA-based data from Mendes et al. [7]. Different lower case letters indicate significant differences $(P$ $<0.05$ ) within DNA-based data, while upper case letters indicate differences within RNA-based data. c Network co-occurrence analysis of microbial communities in the rhizosphere of two common bean cultivars with contrasting levels of resistance to $F$. oxysporum. Each node represents a microbial phylotype (Bacteria or Archaea) at genus level.

Within this family, the genus Paenibacillus was found abundantly in the rhizosphere of the Fox-resistant cultivar (Supplementary Fig. 1). This genus is widely distributed in the environment and known as an antagonist of soil-borne pathogens, including Fusarium [9-11]. Comparing these results with those from our previous DNA-based analyses [7], we found the same patterns/dynamics for all bacterial families and functions detected in both studies (Fig. 2a). Interestingly, we detected in both datasets (DNA and RNA) a higher abundance of bacterial groups belonging to the order Bacillales and the family Geodermatophilaceae in the
A connection stands for SparCC correlation with a magnitude $>0.7$ (positive correlation-blue edges) or $<-0.7$ (negative correlation-red edges) and statistically significant $(P<0.01)$. The size of each node is proportional to the number of connections (that is, degree). Each node was labeled at the phylum level, except for Proteobacteria phylum, shown at the class level. Rs $=$ Fox -resistant cultivar; $\mathrm{Sc}=$ susceptible cultivar (color figure online)

Fox-resistant cultivar when compared with the susceptible cultivar. Species of Bacillales are known for the production of antimicrobial compounds such as bacteriocins, nonribosomally synthesized peptides (NRPs), polyketides (PKs) and lipopeptides (LPs) [12]. Members of Geodermatophilaceae play a vital role in several biogeochemical processes and are characterized as plant growthpromoting rhizobacteria [13]. Our data also revealed that functions related to the metabolism of nitrogen, phosphorus, iron, nucleotides sugars and transcriptional regulation were significantly more expressed in the rhizosphere of the 
Fox-resistant cultivar (Fig. 1d and Supplementary Tables 2 and 3). A higher abundance of genes related to nutrient metabolism could promote plant growth and health, and healthier plants are more prone to fend off pathogen invasions [14]. Also, the resistant cultivar presented more expression of cell motility and chemotaxis genes, which functions are important for the assembly of the rhizosphere microbiome, as they propel microbial populations to available resources [15]. This ability to move also provides a competitive advantage in the rhizosphere, thereby hampering pathogen invasion.

Further exploration of the data revealed higher expression of genes involved in phenazine and colicin $\mathrm{V}$ biosynthesis in the Fox-resistant rhizosphere microbiome (Fig. 1d). Phenazine antibiotics have strong activity against F. oxysporum in several crops and contribute to Fusariumwilt suppressiveness of soils [16]. Colicin V (colV) is a secreted peptide antibiotic capable to kill bacterial cells, thereby reducing competition for nutrients [17]. In a recent study, the production of colV by an Enterobacter sp. strain was related to the suppression of Fusarium wilt in finger millet [18]. Interestingly, genes involved in the biosynthesis of these two 'anti-Fusarium' compounds were also found more abundantly in the rhizosphere of the Fox-resistant bean based on the metagenome data (Fig. 2b). Summarizing, our results suggest that the rhizosphere of the Foxresistant cultivar is enriched with beneficial taxa and functional traits that help the plant to fend off the pathogen. This hypothesis is further supported by the results of the cooccurrence network analysis based on the active community, which showed a more complex network for the Foxresistant cultivar, based on the high number of correlations, high modularity, small diameter, and low average path length (Fig. 2c and Supplementary Table 4). A more complex network has been proposed to better resist pathogen invasions, because of the higher number of species interactions and intensified competition for niche space [15, 19]. To infect root tissue, the fungi have to compete with members of the rhizosphere microbiome for nutrients and microsites [5]. Using the metrics of the network topology we also identified the genus Paenibacillus as key taxon in the Fox-resistant co-occurrence network with high betweenness centrality and high correlations, which can be interpreted as keystone species within a community (Supplementary Table 5). As discussed above, this bacterial genus is widely known for its activity against Fusarium. A pathogen invader may manage to displace the key taxa and collapse the network structure [20]. For example, it was demonstrated that $F$. oxysporum produces fusaric acid, which down regulates the production of the antibiotic compound DAPG in Pseudomonas, a key factor for its antagonistic activity in rhizosphere [21]. Although we have identified a key taxon in the network of the Fox-resistant cultivar, the microbiome is more complex and dynamic. Several other microorganisms that can confer suppressiveness have been found among other bacterial phyla, such as Proteobacteria and Firmicutes [22]. The putative beneficial effects of these and other identified bacterial taxa will be subject of future studies.

In light of our results, we suggest that breeding for resistance in common bean have selected a specific active microbial community which presented high expression of beneficial microbial traits that may complement disease protection in addition to the resistance traits of the plant itself. Our study reinforces the importance of understanding the processes of microbiome assembly in the rhizosphere, where the identification of microbial groups and traits related to pathogen suppression could help the future development of plant breeding to select for plant traits that enrich and activate beneficial microbial groups and genes that will protect plants against root pathogens.

Acknowledgements This study was supported by Fundação de Amparo à Pesquisa do Estado de São Paulo (FAPESP 2014/03217-3, 2015/00251-9) and Coordenação de Aperfeiçoamento de Pessoal de Nível Superior (CAPES 88887.185941/2018-00). Publication 6561 of the Netherlands Institute of Ecology (NIOO-KNAW)

\section{Compliance with ethical standards}

Conflict of interest The authors declare that they have no conflict of interest.

\section{References}

1. Mendes R, Garbeva P, Raaijmakers JM. The rhizosphere microbiome: significance of plant beneficial, plant pathogenic, and human pathogenic microorganisms. FEMS Microbiol Rev. 2013;37: 634-63.

2. Bulgarelli D, Garrido-Oter R, Münch PC, Weiman A, Dröge J, Pan Y, et al. Structure and function of the bacterial root microbiota in wild and domesticated barley. Cell Host Microbe. 2015;17: 392-403.

3. Mendes LW, Kuramae EE, Navarrete AA, van Veen JA, Tsai SM. Taxonomical and functional microbial community selection in soybean rhizosphere. ISME J. 2014;8:1577-87.

4. Meena KK, Sorty AM, Bitla UM, Choudhary K, Gupta P, Pareek A, et al. Abiotic stress responses and microbe-mediated mitigation in plants: the omics strategies. Front Plant Sci. 2017;8:172.

5. Chapelle E, Mendes R, Bakker PAH, Raaijmakers JM. Fungal invasion of the rhizosphere microbiome. ISME J. 2016;10:265-8.

6. Lebeis SL, Paredes SH, Lundberg DS, Breakfield N, Gehring J, McDonald M, et al. Salicylic acid modulates colonization of the root microbiome by specific bacterial taxa. Science. 2015;349: $860-4$.

7. Mendes LW, Raaijmakers JM, Hollander M, Mendes R, Tsai SM. Influence of resistance breeding in common bean on rhizosphere microbiome composition and function. ISME J. 2018;12:212-24.

8. Yao H, Wu F. Soil microbial community structure in cucumber rhizosphere of different resistance cultivars to fusarium wilt. FEMS Microbiol Ecol. 2010;72:456-63.

9. Li P, Ma L, Feng YL, Mo MH, Yang FX, Dai HF, et al. Diversity and chemotaxis of soil bacteria with antifungal activity 
against Fusarium wilt of banana. J Ind Microbiol Biotechnol. 2012;39:1495-505.

10. Budi SW, van Tuinen D, Arnould C, Dumas-Gaudot E, Gianinazzi-Pearson V, Gianinazzi S. Hydrolytic enzyme activity of Paenibacillus sp. strain B2 and effects of the antagonistic bacterium on cell integrity of two soil-borne pathogenic fungi. Appl Soil Ecol. 2000;15:191-9.

11. Dijksterhuis J, Sanders M, Gorris LG, Smid EJ. Antibiosis plays a role in the context of direct interaction during antagonism of Paenibacillus polymyxa towards Fusarium oxysporum. J Appl Microbiol. 1999;86:13-21.

12. Zhao X, Kuipers OP. Identification and classification of known and putative antimicrobial compounds produced by a wide variety of Bacillales species. BMC Genom. 2016;17:882.

13. Xiao X, Fan M, Wang E, Chen W, Wei G. Interactions of plant growth-promoting rhizobacteria and soil factors in two leguminous plants. Appl Microbiol Biotechnol. 2017;101:8485-97.

14. Spann TM, Schumann AW. The role of plant nutrients in disease development with emphasis on citrus and huanglongbing. Proc Flor Stat Hortic Soc. 2009;122:169-71.

15. Mallon CA, van Elsas JD, Salles JF. Microbial invasions: the process, patterns, and mechanisms. Trends Microbiol. 2015;23: 719-29.

16. Mazurier S, Corberand $\mathrm{T}$, Lemanceau $\mathrm{P}$, Raaijmakers JM. Phenazine antibiotics produced by fluorescent pseudomonads contribute to natural soil suppressiveness to Fusarium wilt. ISME J. 2009;3:977-91.

17. Gérard F, Pradel N, Wu L-F. Bactericidal activity of Colicin V Is mediated by an inner membrane protein, $\mathrm{SdaC}$, of Escherichia coli. J Bacteriol. 2005;187:1945-50.

18. Mousa WK, Shearer C, Limay-Rios V, Ettinger CL, Eisen JA, Raizada MN. Root-hair endophyte stacking in finger millet creates a physicochemical barrier to trap the fungal pathogen Fusarium graminearum. Nat Microbiol. 2016;1:16167.

19. Wei Z, Yang T, Friman V-P, Xu Y, Shen Q, Jousset A. Trophic network architecture of root-associated bacterial communities determines pathogen invasion and plant health. Nat Comm. 2015;6:8413.

20. Albrecht M, Padrón B, Bartomeus I, Traveset A. Consequences of plant invasions on compartmentalization and species' roles in plant-pollinator networks. Proc Biol Sci. 2014;281:20140773.

21. Notz R, Maurhofer M, Dubach H, Haas D, Défago G. Fusaric acid-producing strains of Fusarium oxysporum alter 2,4-diacetylphloroglucinol biosynthetic gene expression in Pseudomonas fluorescens CHA0 in vitro and in the rhizosphere of wheat. Appl Environ Microbiol. 2002;68:2229-35.

22. Raaijmakers JM, Paulitz TC, Steinberg C, Alabouvette C, Moënne-Loccoz Y. The rhizosphere: a playground and battlefield for soilborne pathogens and beneficial microorganisms. Plant Soil. 2009;321:341-61. 